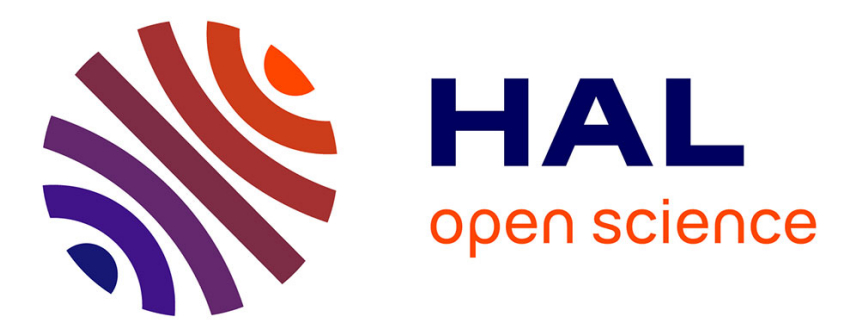

\title{
The influence of phosphorus content on magnetic anisotropy in ferromagnetic (Ga, Mn) (As, P)/GaAs thin films
}

M. Yahyaoui, K. Boujdaria, M. Cubukcu, Christophe Testelin, Catherine

Gourdon

\section{To cite this version:}

M. Yahyaoui, K. Boujdaria, M. Cubukcu, Christophe Testelin, Catherine Gourdon. The influence of phosphorus content on magnetic anisotropy in ferromagnetic (Ga, Mn) (As, P)/GaAs thin films. Journal of Physics: Condensed Matter, 2013, 25 (34), pp.346001. 10.1088/0953-8984/25/34/346001 . hal-01229173

\section{HAL Id: hal-01229173 \\ https://hal.science/hal-01229173}

Submitted on 10 Dec 2015

HAL is a multi-disciplinary open access archive for the deposit and dissemination of scientific research documents, whether they are published or not. The documents may come from teaching and research institutions in France or abroad, or from public or private research centers.
L'archive ouverte pluridisciplinaire HAL, est destinée au dépôt et à la diffusion de documents scientifiques de niveau recherche, publiés ou non, émanant des établissements d'enseignement et de recherche français ou étrangers, des laboratoires publics ou privés. 


\title{
The influence of phosphorus content on magnetic anisotropy in ferromagnetic $(\mathrm{Ga}, \mathrm{Mn})(\mathrm{As}, \mathrm{P}) / \mathrm{GaAs}$ thin films
}

\author{
M. Yahyaoui and K. Boujdaria* \\ Laboratoire de Physique des Matériaux: Structures et Propriétés, \\ Faculté des Sciences de Bizerte, Université de Carthage, 7021 Zarzouna, Bizerte, Tunisia \\ M. Cubukcu, ${ }^{\dagger}$ C. Testelin, and C. Gourdon \\ Institut des Nanosciences de Paris, Université Pierre et Marie Curie, \\ CNRS-UMR7588, Paris, F-75005 France
}

(Dated: June 6, 2013)

\begin{abstract}
The magnetic anisotropy of the ferromagnetic semiconductor (Ga,Mn)(As,P) is studied in a material-specific microscopic k.p approach. We calculate the electronic energy band structure of $(\mathrm{Ga}, \mathrm{Mn})(\mathrm{As}, \mathrm{P})$ quaternary ferromagnetic alloys using a 40-band k.p model and taking into account the s,p-d exchange interaction and the strain of the $(\mathrm{Ga}, \mathrm{Mn})(\mathrm{As}, \mathrm{P})$ layer on a GaAs substrate. We determine the variations of the carrier effective masses in the strained $(\mathrm{Ga}, \mathrm{Mn})(\mathrm{As}, \mathrm{P}) / \mathrm{GaAs}$ system. The magnetic anisotropy constants obtained from our simulations using a mean-field model are compared with the experimental ones determined by ferromagnetic resonance spectroscopy on a set of samples with constant Manganese concentration and varying Phosphorus concentration. An excellent quantitative agreement between experiment and theory is found for the uniaxial outof-plane and cubic-in-plane anisotropy parameters.

PACS numbers: 75.50.Pp, 75.30.Gw, 75.70.Ak, 73.20.At
\end{abstract}




\section{INTRODUCTION}

For the past two decades, research in diluted magnetic semiconductors (DMS) has attracted tremendous interest because of their great potential for different spintronic applied devices $^{1-3}$. Ferromagnetic semiconductors (SC) might combine storage, detection, logic and communication capabilities on a single chip to produce multifunctional devices such as magnetic sensors, spin injectors, and magnetic memories. Among them, the $\mathrm{Ga}_{1-x} \mathrm{Mn}_{x} \mathrm{As}$ compounds have received a lot of attention (see [4] and references therein). These alloys exhibit many interesting magnetic properties, such as a relatively high Curie temperature ${ }^{5}$ and complex magnetic anisotropy (MA) that determines the easy magnetization axes in the absence of external field ${ }^{6-10}$. Since information is stored in the direction of magnetization in magnetic storage devices, switching between several easy axes and precession of the magnetization have been considered for information storage and/or processing ${ }^{11,12}$.

As a consequence of the semiconductor band structure (BS) of $\mathrm{Ga}_{1-x} \mathrm{Mn}_{x} \mathrm{As}$, the MA is strongly dependent on Mn and carrier concentrations, temperature, and strain ${ }^{10,13,14}$. This dependence allows the use of various static or quasi-static methods to intentionally manipulate the magnetic easy axes. The control of the desired direction of the easy magnetization (in-plane or out-of-plane) has been achieved by (i) changing Mn concentration ${ }^{6,15}$, (ii) changing carrier concentration by hydrogenation ${ }^{16}$, (iii) varying temperature $^{6,17}$, (iv) choosing different substrates and therefore different growth-induced strain in the magnetic layer ${ }^{17,18}$, (v) bonding the sample to a piezo-transducer and applying a voltage to change the strain ${ }^{19-21}$, (vi) inducing strain relaxation by lithography patterning ${ }^{12,22}$. Furthermore dynamical modification of the MA by strain was achieved by using short strain pulses to induce a transient change of the MA in order to launch magnetization precession ${ }^{23,24}$.

The prediction of MA under various conditions of strain, Mn and carrier concentrations and its comparison to experimental results has received a lot of attention in $\mathrm{Ga}_{1-x} \mathrm{Mn}_{x} \mathrm{As}$. Strain control of MA in $\mathrm{Ga}_{1-x} \mathrm{Mn}_{x} \mathrm{As}_{1-y} \mathrm{P}_{y}$, the focus of this paper, has so far been explored much less extensively. Introducing a few percent of phosphorus at the arsenic sites in $\mathrm{Ga}_{1-x} \mathrm{Mn}_{x}$ As layers modifies the epitaxial strain continuously from compressive to tensile independently of the $\mathrm{Mn}$ doping level ${ }^{25-27}$. One can thereby tune the magnetic easy axis from in-plane to out-of-plane simply by increasing the phosphorus content. Moreover, these layers contain much less defects than $\mathrm{Ga}_{1-x} \mathrm{Mn}_{x}$ As layers designed to produce normal-to- 
plane magnetic easy axis, such as $\mathrm{Ga}_{1-x} \mathrm{Mn}_{x} \mathrm{As}$ grown on a relaxed (Ga,In)As buffer layer. Indeed, the self-organization of magnetic domains in periodic patterns, impossible to reach in $(\mathrm{Ga}, \mathrm{Mn}) \mathrm{As} /(\mathrm{Ga}, \mathrm{In}) \mathrm{As}$, was observed in $(\mathrm{Ga}, \mathrm{Mn})(\mathrm{As}, \mathrm{P}) / \mathrm{GaAs}^{28}$. These quaternary layers also have other important advantages over $\mathrm{Ga}_{1-x} \mathrm{Mn}_{x} \mathrm{As}$. For example, it has been predicted that the decrease in the lattice constant of $\mathrm{Ga}_{1-x} \mathrm{Mn}_{x} \mathrm{As}_{1-y} \mathrm{P}_{y}$ should lead to an increase in the Curie temperature and the exchange integral $\mathrm{J}_{p d}{ }^{29}$, which was partly confirmed by experiments $^{28}$.

As far as we know, although several works were devoted to the study of $\mathrm{Ga}_{1-x} \mathrm{Mn}_{x} \mathrm{As}_{1-y} \mathrm{P}_{y}{ }^{25-31}$, the phosphorus composition dependence of the $\mathrm{Ga}_{1-x} \mathrm{Mn}_{x} \mathrm{As}_{1-y} \mathrm{P}_{y}$ band parameters and the link between MA and the band structure has not been theoretically investigated. Let us note that one cannot simply map onto $\mathrm{Ga}_{1-x} \mathrm{Mn}_{x} \mathrm{As}_{1-y} \mathrm{P}_{y}$ the calculations performed for $\mathrm{Ga}_{1-x} \mathrm{Mn}_{x}$ As. These generally use the band parameters of GaAs. Here one needs to input the band structure of the $\mathrm{GaAs}_{1-y} \mathrm{P}_{y}$ alloy.

In a recent article $^{32}$, we have investigated the effect of epitaxial strain on the MA in a ferromagnetic $(\mathrm{Ga}, \mathrm{Mn})(\mathrm{As}, \mathrm{P})$ thin film with a typical composition of $\mathrm{Mn} 7 \%$ and $\mathrm{P} 7 \%$. One of the remaining open questions is the influence of phosphorus fraction on the electronic and magnetic properties of $(\mathrm{Ga}, \mathrm{Mn})(\mathrm{As}, \mathrm{P})$.

This paper is organized as follows. Section II presents the calculation of the energy BS and band parameters of strained $\mathrm{Ga}_{1-x} \mathrm{Mn}_{x} \mathrm{As}_{1-y} \mathrm{P}_{y} / \mathrm{GaAs}$ within a 40-band k.p model using the mean-field Zener model ${ }^{13}$. The influence of subsequent approximations to the electronic structure (6-band $\mathbf{k} \cdot \mathbf{p}$ model) is discussed. The carrier masses and Luttinger parameters are determined as a function of the Phosphorus fraction. In Sec. III, we present a quantitative comparison of the anisotropy parameters obtained from ferromagnetic resonance (FMR) experiments on a set of samples with similar Mn concentration and varying $\mathrm{P}$ concentration with our model calculation of the MA using the BS determined in Sec. II. The final section of this work is devoted to the conclusion and perspectives. 


\section{BAND STRUCTURE}

\section{A. Methodology}

In order to obtain the dependence of the MA as a function of phosphorus fraction, the initial step is the BS calculation of $\mathrm{Ga}_{1-x} \mathrm{Mn}_{x} \mathrm{Ass}_{1-y} \mathrm{P}_{y}$ bulk SCs using $\mathbf{k} \cdot \mathbf{p}$ theory. For this purpose, we use a 40-band $\mathbf{k} \cdot \mathbf{p}$ Hamiltonian first introduced by Saïdi et al. ${ }^{33}$. It takes into account the (s, p, d) levels both in the valence bands (VBs) and conduction bands (CBs). We denote by $\mathbf{H}_{40}$ the Hamiltonian matrix. Our $40 \times 40$ k.p Hamiltonian involves matrix elements between the ten $\left(\Gamma_{3 d}^{C}+\Gamma_{5 d}^{C}\right)$, ten $\left(\Gamma_{3 d}^{V}+\Gamma_{5 d}^{V}\right)$, six $\Gamma_{5 C}$, six $\Gamma_{5 V}$, two $\Gamma_{1 C}$, two $\Gamma_{1 V}$, two $\Gamma_{1 u}$, and two $\Gamma_{1 q}$ wavefunctions. A full expression of the $40 \times 40$ Hamiltonian matrix is explicitly given in [34]. This model depends on $10 \Gamma$-centered eigenvalues, 18 spin-orbit coupling coefficients, and 30 matrix elements. In Ref. [33], the energy gaps, the momentum matrix elements, and the spin-orbit interaction coefficients used within this model are given in detailed form.

When the crystal is strained, additional terms must be introduced in the k.p Hamiltonian ${ }^{35-37}$. In the case of a biaxially strained $\mathrm{Ga}_{1-x} \mathrm{Mn}_{x} \mathrm{As}_{1-y} \mathrm{P}_{y}$ layer grown on a [001]oriented cubic GaAs buffer, the biaxial strain tensor can be written as a diagonal tensor with elements $\epsilon_{x x}, \epsilon_{y y}, \epsilon_{z z}^{10,13}$.

In this work, we have adopted the notations of Glunk et al. ${ }^{9}$ for the relaxed lattice parameter and strain. Hence, $\epsilon_{z z}=\frac{a_{\perp}-a_{r e l}}{a_{r e l}}, \epsilon_{x x}=\epsilon_{y y}$ and $\epsilon_{z z}=-2 \frac{C_{12}}{C_{11}} \epsilon_{x x}$. The relaxed lattice parameter $a_{r e l}$ of a biaxially strained layer on [001]-oriented substrate is given by

$$
a_{r e l}=\frac{2 C_{12}}{C_{11}+2 C_{12}} a_{/ /}+\frac{C_{11}}{C_{11}+2 C_{12}} a_{\perp}
$$

where $\mathrm{a}_{/ /}$and $\mathrm{a}_{\perp}$ are the lateral and vertical lattice parameters of the layer. $\mathrm{C}_{11}$ and $\mathrm{C}_{12}$ are elastic stiffness constants of bulk $\mathrm{GaAs}_{1-y} \mathrm{P}_{y}$. They are related to the phosphorus content $y$ by (in $\left.10^{11} \mathrm{dyn} \mathrm{cm}^{-2}\right)^{38}$

$$
\begin{aligned}
& C_{11}=12.37+2.1895 y+0.2845 y^{2} \\
& C_{12}=5.26+0.9310 y+0.1210 y^{2} .
\end{aligned}
$$


Using Eq. 1, the vertical strain $\epsilon_{z z}$ can be written as

$$
\epsilon_{z z}=\operatorname{lm} \frac{1-\frac{C_{11}}{\left(C_{11}+2 C_{12}\right)}}{1+\frac{C_{11}}{\left(C_{11}+2 C_{12}\right)} l m},
$$

where $l m=\frac{\left(a_{\perp}-a_{/ /}\right)}{a_{/ /}}$is the lattice mismatch determined by high resolution x-ray diffraction.

The characteristics of the samples studied in this paper are summarized in Table I. The seven $\mathrm{Ga}_{0.93} \mathrm{Mn}_{0.07} \mathrm{As}_{1-y} \mathrm{P}_{y}$ samples are selected in order to compare layers with the same nominal Mn concentration (7\%) and different phosphorus concentrations. The samples were grown by low temperature molecular beam epitaxy on $\mathrm{GaAs}(001)$ substrates. The Mn and $\mathrm{P}$ concentrations were calibrated using Vegard's law, based on the lattice constants of a series of $\mathrm{GaAs}_{1-y} \mathrm{P}_{y}$ references samples grown under the same conditions. After growth, the samples were systematically annealed under nitrogen atmosphere at $250{ }^{\circ} \mathrm{C}$ for 1 hour ${ }^{25}$. The data listed in Table I clearly show that the strain changes from compressive $\left(\epsilon_{z z}>0\right)$ to tensile $\left(\epsilon_{z z}<0\right)$ when the phosphorus composition increases in the $50 \mathrm{~nm}$ thick $\mathrm{Ga}_{1-x} \mathrm{Mn}_{x} \mathrm{As}_{1-y} \mathrm{P}_{y}$ epilayer.

The Bir-Pikus $\mathbf{H}_{S}$ Hamiltonian matrix used in this work is given explicitly in Ref. 34 . Because the influence of strain on p-type $\mathrm{CB}$ is unknown, we have taken the p-type $\mathrm{CB}$ hydrostatic deformation potential $\left(a_{\Gamma_{5} C}\right)$ and the p-type $\mathrm{CB}$ shear deformation potential $\left(b_{\Gamma_{5 C}}\right)$ equal to zero. Moreover, we do not use the Bir-Pikus Hamiltonian for the s-type $\mathrm{VB}$, which is at about $-12.5 \mathrm{meV}$ from the top of the $\mathrm{VB}$, because (i) no parameters are available for this band and (ii) its influence on the $\mathrm{CB}$ and on the VB of interest is very small. Thus, we consider $a_{\Gamma_{1 V}}=0$. In the same way there is no need to apply the strain to the d-type VBs and CBs.

The presence of $\mathrm{Mn}$ ions in $\mathrm{Ga}_{1-x} \mathrm{Mn}_{x} \mathrm{As}_{1-y} \mathrm{P}_{y}$ is taken into account by including the $s, p$ $d$ exchange interactions. Hence, $\mathbf{H}_{e x c}=\mathbf{H}_{p d}+\mathbf{H}_{s d}$ describes the exchange coupling between the localized $d$-electrons and the delocalized holes $(p)$ and electrons $(s) . \mathbf{H}_{p d}$ describes the $p$ - $d$ hybridization, which yields an effective exchange interaction between the hole spin $\mathbf{s}$ and the Mn spin $\mathbf{S}(S=5 / 2)$ carrying a magnetic moment $-S g \mu_{B}$, where $g$ is the Landé factor and $\mu_{B}$ the Bohr magneton. The exchange interaction can be further simplified by the meanfield approximation where the virtual crystal approximation is made ${ }^{2,13}$. The conduction and valence bands exchange splittings are obtained from the exchange energies $N_{0} \alpha$ and $N_{0} \beta$ where $N_{0}$ is the concentration of cation sites and $\alpha$ and $\beta$ are the $s-d$, and $p-d$ exchange 
integrals, respectively. In order to obtain the values of $N_{0} \beta$ we assume $N_{0} \sim 4 / a_{\text {rel }}^{3}$ and $\beta=-$ $54.5 \mathrm{meV} \mathrm{nm}^{3}$. $a_{\text {rel }}$ varies from 5.6661 to $5.6359 \AA$ and $N_{0} \beta$ from -1.20 to $-1.22 \mathrm{eV}$ for $y$ from 0 to $11.3 \%$. Since there is no report concerning experimental or theoretical determination of the $s$ - $d$ exchange energy $N_{0} \alpha$ the value $200 \mathrm{meV}$, as observed for II-VI diluted magnetic $\mathrm{SCs}^{39}$, has been adopted in this work. Owing to the presence of $\mathrm{Mn}$ atoms in interstitial sites the effective concentration of $\mathrm{Mn}$ atoms participating in the ferromagnetism is lower than the total one ${ }^{40}$. $x_{\text {eff }}$ is deduced from the low-temperature saturation magnetization $\mathrm{M}_{S}$ as $x_{e f f}=M_{S} /\left(N_{0} S g \mu_{B}\right)$. Table I clearly shows that only about $50 \%$ of Mn atoms contribute to the magnetization for all samples, revealing that the incorporation of $\mathrm{Mn}$ in interstitial sites is not modified by alloying with phosphorus. One should also note that, in the Zener model, the exchange splitting of the valence subbands $B_{G}=A_{F} \beta M_{S} / 6 g \mu_{B}$ is an important parameter for the BS and the MA. The values used in our calculations are given in Table I. The $A_{F}$ parameter describes the enhancement of spin density of states by the carrier-carrier interactions. The best fit with experimental results is obtained with $A_{F}=1.4$ (close to the value 1.2 used in [13]).

According to the k.p effective Hamiltonian theory, the total Hamiltonian $\mathbf{H}$ of the system used in this work has the general form: $\mathbf{H}=\mathbf{H}_{\mathbf{k p}}+\mathbf{H}_{S}+\mathbf{H}_{\text {exc }}$. Let us note that we have not taken into account the band gap renormalization arising from the high density of carriers in the (III-Mn)V compounds since we are interested in MA and not in optical transitions.

\section{B. Results}

In this section we present and discuss the results of the application of our $\mathbf{k} . \mathbf{p}$ model to the electronic structure calculations for bulk $\mathrm{Ga}_{1-x} \mathrm{Mn}_{x} \mathrm{As}_{1-y} \mathrm{P}_{y} \mathrm{SC}$. All these calculations were carried out at the relaxed lattice constant. An example of the BS calculated with the full Hamiltonian $\mathbf{H}$ for $x_{\text {eff }}=0.0369$ and $y=0$ is shown in Fig. 1. The electronic BS of this compounds shows features similar to that of bulk GaAs. The band diagram is well reproduced on a width of about $18 \mathrm{eV}$. The extended k.p method is known to be valid up to $6 \mathrm{eV}$ above and $12 \mathrm{eV}$ below the top of the $\mathrm{VB}$ in four directions namely $\Gamma X, \Gamma L, \Gamma K$ and $X U$.

One of the important effect of the strain and exchange is observable in the VB near- $\Gamma$ region. In Figs 2a-2c, we show a magnified BS near $k=0$, for three different phosphorus 
fraction (a) $y=0 \%$, (b) $y=3.4 \%$, and (c) $y=11.3 \%$, corresponding to the regime of compressive, zero, and tensile strain, respectively. It is clear that the degeneracy between heavy-holes (HHs) and light-holes (LHs) at the center of the Brillouin zone is lifted. Moreover, the band energies shift evidently near the $\Gamma$ point, while there is a smaller shift on other VB regions. The influence of the $\mathrm{P}$ concentration is mostly evidenced along the [111] direction (Fig. 2(c)).

Generally, due to the presence of the strain, the BS of SCs is altered, which changes the lattice constant and reduces the symmetry of the crystal. It also modifies the energy gaps, and lifts degeneracy. The influence of subsequent approximations on the electronic structure is considered. In Fig. 2(d) we show the comparison of the VB dispersion obtained from the 40-band model (solid line) and that from the 6-band model (dashed line) for the case of $\mathrm{Ga}_{0.9631} \mathrm{Mn}_{0.0369} \mathrm{As}$ over the [001] and [111] directions. This figure shows a satisfactorily agreement between the two models only up to $k \sim 0.1 \AA^{-1}$ measured from the $\Gamma$ edge. For a Fermi energy in $(\mathrm{Ga}, \mathrm{Mn})$ As up to $\approx 100 \mathrm{meV}$ the 6 -band model is probably sufficient. For higher Fermi energies (doping level above $\approx 510^{20} \mathrm{~cm}^{-3}$ ) the deviation of the 6-band k.p model BS from the real BS might lead to an inaccurate estimation of the MA. Let us emphasize that within the 6-band model, one needs to specify the Luttinger parameters in order to take into account the interaction of the VB with the remote bands. This can be achieved for $(\mathrm{Ga}, \mathrm{Mn})$ As by taking the parameters of GaAs but these parameters are not available for $(\mathrm{Ga}, \mathrm{Mn})(\mathrm{As}, \mathrm{P})$. In the framework of the 40-band model the coupling between bands is described in terms of the matrix elements and band edges that are deduced from the experiments. Once the band structure is well reproduced with the 40-band model, we then calculate the Luttinger parameters ${ }^{33}$ which can be used within the 6 -band model. This is an important advantage of the 40-band model compared to the 6-band model.

To investigate the effect of phosphorus fraction on the electronic properties of alloys, we also performed calculations of energy gaps at high-symmetry points in the Brillouin zone. The band gap energy at $\Gamma$ point, $\mathbf{E}_{g}^{\Gamma}$, is defined as the difference between the lowest conduction band and the highest VB in the presence of strain and exchange. The variation of $\mathbf{E}_{g}^{\Gamma}$ as a function of the phosphorus content in the quaternary alloy layers $\mathrm{Ga}_{1-x} \mathrm{Mn}_{x} \mathrm{As}_{1-y} \mathrm{P}_{y} / \mathrm{GaAs}$ is displayed in Fig. 3. This variation results from four contributions: (i) the increase of the gap of the bulk $\mathrm{Ga}(\mathrm{As}, \mathrm{P})$ with $\mathrm{P}$ concentration (alloying effect), (ii) the down-shift of the gap resulting from the exchange splitting of the valence and conduction bands (almost constant 
here since $N_{0} \beta$ and $x_{\text {eff }}$ have small variations with $y$ ), (iii) the effect of the hydrostatic part of the strain that increases the gap for compressive strain and decreases it for the tensile one, thus opposing the alloying effect, and finally (iv) the effect of the biaxial strain on the VB that gives a variation similar to the hydrostatic component but with a smaller amplitude. The increase in the gap seen in Fig. 3 results from the alloying effect (i). The plateau near $y \approx 4 \%$ results from the cancellation of strain contributions (iii) and (iv) at zero strain together with a slightly increased exchange down-shift (13\% larger for $y=6 \%$ than for $0 \%$ and $11.3 \%)$.

We proceed to understand the role of phosphorus fraction on the effective masses and Luttinger parameters. For that purpose we calculate the effective masses for s-type CB $\left(m_{e}\left(\Gamma_{6 C}\right)\right)$ and for p-type $\operatorname{VBs}\left(m_{h h}, m_{\ell h}\right)$ using the total Hamiltonian $\mathbf{H} . m_{e}\left(\Gamma_{6 C}\right)$ was obtained from the second derivative of s-type $\mathrm{CB}$ energy with respect to the wave vector around the $\Gamma$ point. As can be seen in Fig. 4a, the electron effective mass of quaternary $\mathrm{Ga}_{1-x} \mathrm{Mn}_{x} \mathrm{As}_{1-y} \mathrm{P}_{y}$ exhibits a non-monotonous dependence on the phosphorus composition. Indeed, $m_{e}\left(\Gamma_{6 C}\right)$ increases mildly with decreasing compressive biaxial strain, while it decreases rapidly with tensile strain. This non-monotonous behavior reflects a competition between alloying and strain effects. Let us note that the variation of the Mn composition has no effect on the masses since the exchange splitting does not change the band curvature.

Generally speaking, these observations are consistent with previous experiments and theoretical study of other bulk SCs ${ }^{41}$. In Figs 4b-4c, we show the effective masses for both the $\mathrm{HH}$ and LH bands along [001], [110] and [111] directions as a function of phosphorus fraction. $m_{h h}$ and $m_{\ell h}$ have the same behavior along all three directions. Similarly to the electron mass the $\mathrm{HH}$ mass exhibits a non-monotonous behavior with $\mathrm{P}$ concentration. It decreases slightly under decreasing compressive strain and increases mildly with tensile strain. The LH mass decreases monotonously with increasing $y$ but the slope of $m_{\ell h}(y)$ changes when going from compressive to tensile strain. Note finally that the $m_{h h}$ effective mass is the largest along [111] direction, while $m_{\ell h}$ is the largest along the [001] direction.

An accurate description of the VB effective masses makes our results reliable to calculate the Luttinger parameters $\gamma_{\ell}(\ell=1,2,3)$ from the following expression ${ }^{42}$ 


$$
\left\{\begin{array}{c}
\gamma_{1}=1 / 2\left(1 / m_{l h}^{001}+1 / m_{h h}^{001}\right) \\
\gamma_{2}=1 / 4\left(1 / m_{l h}^{001}-1 / m_{h h}^{001}\right) \\
\gamma_{3}=1 / 4\left(1 / m_{l h}^{001}+1 / m_{h h}^{001}\right)-2 / m_{h h}^{111}
\end{array}\right.
$$

and shows that the behavior of the Luttinger parameters is a consequence of the competition between the alloying effect and the strain effect. In Fig. 5, we show the dependence of the Luttinger parameters on $y$ and compare them to data from the literature for unstrained $\mathrm{GaAs}_{1-y} \mathrm{P}_{y}$ alloys. The full lines show the $y$-variation obtained by a linear interpolation between the GaAs and GaP values ${ }^{43}$. The decrease in $\gamma_{\ell}(\ell=1,2,3)$ with increasing $y$ (full lines) can be explained by an important alloying effect. For our calculated values (symbols), the effect of strain differs depending on the regime of strain considered. Indeed, comparatively to the unstrained case, the compressive strain leads to a decrease of the Luttinger parameters while the tensile strain leads to an increase of $\gamma_{\ell}$.

\section{MAGNETIC ANISOTROPY}

MA has focused much attention because it is essential not only for the fundamental understanding of the microscopic origins of ferromagnetism in DMS, but also it is of central importance for applications of these materials, since it determines the easy axis of magnetization, the anisotropy fields and the magnetic domain wall width and specific energy. Let us recall that the MA is the dependence of the free energy density $F$ of a magnetic system on the orientation of the magnetization direction $\mathbf{m}=(\mathbf{M} / M)$ with respect to symmetry axes. Recent studies of the ferromagnetic phase in $\mathrm{Ga}_{1-x} \mathrm{Mn}_{x} \mathrm{As}_{1-y} \mathrm{P}_{y}$ epilayers demonstrated the existence of rich $\mathrm{MA}^{25,26,30}$. In order to provide a concise description of the MA, the normalized quantity $F_{M}=F / M$ is considered instead of $F$. As given in Refs. [44,45,46], there are three contributions to $F_{M}$ : (i) the contribution $F_{M, 1}(\mathbf{m})$ that arises from spin-orbit coupling in the VB in a cubic crystal with exchange and biaxial strain, (ii) the contributions $F_{M, 2}(\mathbf{m})$ caused by the demagnetization field and a uniaxial in-plane contribution along [1]10], whose microscopic origin is still under debate ${ }^{6,49-52}$, and (iii) the contribution $F_{M, 3}(\mathbf{m})$ from the single-ion anisotropy ${ }^{46,47}$, which is small and usually neglected. The free-energy functional $F_{M}$ is then given by $F_{M}(\mathbf{m})=F_{M, 1}(\mathbf{m})+F_{M, 2}(\mathbf{m})+F_{M, 3}(\mathbf{m})$. We consider a phenomenological description of the free energy in terms of anisotropy constants and the direction cosines 
of the magnetization vector $m_{x}, m_{y}$, and $m_{z}$ with respect to the main crystallographic directions $\langle 100\rangle$. We restrict our calculations to zero temperature and zero magnetic field. In this approximation and taking only up to the fourth order term for the $m_{i}$ components, the free-energy density for [001]-oriented layers can be written as

$F_{M}(\mathbf{m})=B_{2 \perp} m_{z}^{2}+B_{4 / /}\left(m_{x}^{4}+m_{y}^{4}\right)+B_{4 \perp} m_{z}^{4}+B_{d} m_{z}^{2}+\frac{B_{\overline{1} 10}}{2}\left(m_{x}-m_{y}\right)^{2}+B_{2 \perp}^{s i} m_{z}^{2}+B_{4 / /}^{s i}\left(m_{x}^{4}+m_{y}^{4}\right)+B_{4 \perp}^{s i} m_{z}^{4}$,

where $B_{d}=\frac{\mu_{0} M}{2}$. The first three anisotropy components represent contributions to $F_{M, 1}$. The next two contribute to $F_{M, 2}$, and the last three to $F_{M, 3}$. The parameters $B_{\ell}$ denote the magnetic anisotropy constants. $\left(B_{2 \perp}+B_{2 \perp}^{s i}\right)$ is the perpendicular uniaxial anisotropy field resulting from the biaxial strain introduced during the molecular-beam epitaxy growth of GaMnAs layers on substrates with a different lattice constant. $\left(B_{4 / /}+B_{4 / /}^{s i}\right)$ and $\left(B_{4 \perp}+\right.$ $\left.B_{4 \perp}^{s i}\right)$ are constants representing the in-plane and the perpendicular cubic anisotropy which reflects the non-equivalence of the $\langle 100\rangle$ and $\langle 110\rangle$ directions. In the case of a perfect cubic crystal, the symmetry requires $B_{4 / /}=B_{4 \perp}$. Since growth on a lattice-mismatched substrate introduces tetragonal distortion and breaks the purely cubic symmetry of the crystal lattice, the in-plane and perpendicular cubic anisotropy constants need to be distinguished. $B_{d}$ is the demagnetizing anisotropy field, arising from shape anisotropy. $B_{\overline{1} 10}$ or $B_{2 / /}$ is the in-plane uniaxial anisotropy field that reflects the non-equivalence of [110] and [110] directions.

In our theoretical approach, we have calculated the $F_{M, 1}$ contribution. The numerical procedure to determine the magnetic anisotropy is described in Refs. [9, 32]. Using the BS calculated in Sec. II $F_{1}$ is obtained first by summing over all energy eigenvalues within the four spin-split, HH and LH Fermi surfaces. The resulting energy density is then normalized by the saturation magnetization $M$. We use the sample parameters specified in Table I. Due to the presence of charge compensating defects depending on growth conditions in $\mathrm{Ga}_{1-x} \mathrm{Mn}_{x} \mathrm{As}_{1-y} \mathrm{P}_{y}$ samples, the exact determination of the Fermi level in ferromagnetic $\mathrm{Ga}_{1-x} \mathrm{Mn}_{x} \mathrm{As}_{1-y} \mathrm{P}_{y}$ is difficult. Hence, for reasons of simplicity and lack of data on the hole concentration, we have assumed that the Fermi level has a constant value equal to $-110 \mathrm{meV}$ from the top of the VB for all samples except sample G. In order to justify this approximation, we calculate the hole concentration $p_{\text {theo }}$ for the chosen Fermi energy, and compare it to the estimated hole concentration $p_{\text {est }}$. The values of $p_{\text {est }}$ are obtained by assuming that the reduction of the hole concentration is only due to the presence of interstitial Mn that act as double donors. We have $x_{\text {eff }}=x-2 x_{i}$, where $x_{i}$ is the interstitial 
Mn content. Consequently, the hole concentration is $p_{\text {est }}=N_{0}\left(x-3 x_{i}\right)=N_{0}\left(3 x_{\text {eff }}-x\right) / 2^{53}$. The values of $p_{\text {theo }}$ and $p_{\text {est }}$ are listed in Table II for the studied samples. We can see that taking the Fermi level as constant is a fairly reasonable approximation. Let us note that sample $\mathrm{G}(y=11.3 \%)$ is close to the transition from the metallic to the impurity band conduction regime, as deduced from the temperature dependence of the resistivity. As argued in [2], our model might nevertheless be applicable. Indeed, using a slightly smaller Fermi energy $(-81 \mathrm{meV})$, in agreement with the estimated carrier concentration $p_{\text {est }}$ (Table II), we will see that a good agreement between theoretical and experimental MA fields is obtained.

In order to compare the microscopic theory with the phenomenological description of the MA, we consider the anisotropic part $\Delta F_{M, 1}$ of the $F_{M, 1}$ contribution. When the reference magnetization direction is taken along [100], $\Delta F_{M, 1}$ is given by the following expression

$$
\Delta F_{M, 1}=F_{M, 1}(\mathbf{m})-F_{M, 1}(\mathbf{m}=[100])=B_{2 \perp} m_{z}^{2}+B_{4 / /}\left(m_{x}^{4}+m_{y}^{4}-1\right)+B_{4 \perp} m_{z}^{4} .
$$

For $\mathbf{m}$ rotated in the (001) and in the (010) plane, we can write Eq. 4 as $^{9}$ :

$$
\Delta F_{M, 1}(\varphi)=B_{4 / /}\left(\cos ^{4}(\varphi)+\sin ^{4}(\varphi)-1\right)
$$

and

$$
\Delta F_{M, 1}(\theta)=B_{2 \perp} \cos ^{2}(\theta)+B_{4 / /}\left(\sin ^{4}(\theta)-1\right)+B_{4 \perp} \cos ^{4}(\theta) .
$$

Once $\Delta F_{M, 1}$ is calculated numerically in the microscopic model as a function of the crystalline orientations $(\theta, \varphi)$ for the different phosphorus fractions, the phenomenological description of MA, namely Eqs $(5,6)$, can be fitted to the resulting angular dependences by using $B_{2 \perp}, B_{4 / /}$, and $B_{4 \perp}$ as fit parameters.

The results of the microscopic calculations are shown by full symbols in Figs. 6(a) and 6(b). The full lines show least-squares fits to the calculated data using Eqs. 5 and 6 . We observe that the fit curves are in very good agreement with the microscopic results. This good fit clearly demonstrates that the microscopic theory can be well described by $B_{2 \perp}, B_{4 / /}$, and $B_{4 \perp}$. Therefore a realistic description of the $F_{M, 1}$ part of the free energy only needs terms up to the fourth order in $\mathbf{m}$. We proceed to understand the role of the phosphorus fraction on the magnetic properties of $\mathrm{Ga}_{1-x} \mathrm{Mn}_{x} \mathrm{As}_{1-y} \mathrm{P}_{y}$ quaternary alloys. For 
that purpose we calculate $\Delta F_{M, 1}(\varphi)$ and $\Delta F_{M, 1}(\theta)$ for three representative $\mathrm{P}$ concentrations: $y=11.3 \%$ (tensile strain), $y=3.4 \%$ ( $\approx$ zero strain), and $y=0.0 \%$ (compressive strain), respectively. Fig. 6(a) shows that in $\mathrm{Ga}_{1-x} \mathrm{Mn}_{x} \mathrm{As}_{1-y} \mathrm{P}_{y}$ there is a stronger fourfold cubic in-plane anisotropy constant $B_{4 / /}$ under tensile strain than under compressive strain. Such a behavior has also been shown in the case of $\mathrm{Ga}_{1-x} \mathrm{Mn}_{x} \mathrm{As}$ ferromagnetic $\mathrm{SC}^{9,54}$.

From Fig. 6(b), we observe that in the regime of tensile strain $\Delta F_{M, 1}$ exhibits a minimum for $\mathbf{m}$ oriented along [001] (by symmetry also along [001] ). For compressive strain, the minima occur for $\mathbf{m}$ oriented [100], [100], [010], and [00]. Thus, the theoretical model correctly describes the well-known experimental fact that the magnetically hard axis along [001] in compressively strained layers turns into an easy axis in tensily strained layers as observed in Ref. 26. Note, however, that for the regime of zero strain, the easy axis is not aligned along any high symmetry direction. This result is due to the values approximately equal taken by the cubic anisotropy parameters $\left(B_{4 / /}, B_{4 \perp}\right)$. These observations clearly point out that the easy axis of magnetization is sensitive to the variation of the phosphorus fraction or, in other words, the preferential axes of the magnetization are closely related to the strain conditions.

Applying the phenomenological procedure described above to the whole set of $\mathrm{Ga}_{1-x} \mathrm{Mn}_{x} \mathrm{As}_{1-y} \mathrm{P}_{y} / \mathrm{GaAs}$ layers under study, the anisotropy parameters $B_{2 \perp}, B_{4 / /}$, and $B_{4 \perp}$ were determined as a function of phosphorus fraction.

Let us now consider the single-ion anisotropy contribution $F_{M, 3}$. The uniaxial term $B_{2 \perp}^{s i}$ can be estimated from the D parameter of the single-ion Hamiltonian used in the analysis of electron paramagnetic resonance experiments $(\mathrm{EPR})^{46,47}$ as $B_{2 \perp}^{s i}=\frac{D S}{g \mu_{B}}$. Extrapolating the variation of D obtained from EPR for very low Mn concentrations ${ }^{46}$ would give $B_{2 \perp}^{s i}=9 \mathrm{mT}$ for Sample A. This is $5 \%$ of the value of $B_{2 \perp}$. The $B_{2 \perp}^{s i}$ contribution might even be much smaller since the extrapolation was found to overestimate the $\mathrm{D}$ value by a factor of $\approx 4$ for a concentration as low as $0.0027^{47}$. The value of $\mathrm{D}$ is not known for the same Mn spin state in GaP. However D is proportional to the spin-lattice coupling coefficient $G_{11}$, which is at least 3 times smaller in GaP than in GaAss ${ }^{48}$. Therefore we expect that the $B_{2 \perp}^{s i}$ contribution in $(\mathrm{Ga}, \mathrm{Mn})(\mathrm{As}, \mathrm{P})$ (for the same absolute value of strain) would be even smaller. There might also be a cubic contribution from the single-ion anisotropy. From the $a$ parameter obtained from EPR experiments ${ }^{46,47}$, a $B_{4}^{s i}$ contribution can be estimated as $\frac{a S^{3}}{6 g \mu_{B}}$, which amounts to $4 \mathrm{mT}$. Given the smallness of the single-ion contribution to anisotropy, in the following the 
experimental results will be compared to the carrier contributions $B_{2 \perp}, B_{4 / /}$, and $B_{4 \perp}$.

The experimental magnetic anisotropy constants were obtained by a standard X-band FMR spectroscopy with a $9 \mathrm{GHz}$ microwave source. The angular dependence of the FMR spectra were measured by rotating the static magnetic field in two crystallographic planes: (110) and (001) named "out-of plane" and "in-plane configuration", respectively. These two sets of angular variations of the resonance field enable us to determine the anisotropy constants and associated anisotropy fields ${ }^{44}$.

We have taken the experimental data at $T=4.2 \mathrm{~K}$ in order to compare with our calculations performed at zero temperature. Firstly, we focus on the behavior of $B_{2 \perp}$ parameter. In Fig. $7(\mathrm{a}), B_{2 \perp}$ is plotted versus phosphorus fraction $y$. The microscopic calculations are in very good agreement with experimental data. However, the results of $B_{2 \perp}$ deduced from the microscopic model are found to be slightly different from the experimental data for $y=3.4 \%$ and $y=4.3 \%$. The microscopic model predicts that $B_{2 \perp}$ is linearly proportional to the uniaxial deformation $\epsilon_{z z}$. Indeed, a zero value of the uniaxial out-plane parameter is found for zero strain $(y=3.4 \%)$. However a non-zero value is obtained experimentally. This discrepancy might originate from the experimental difficulty to determine the strain in this range giving a large error bar at zero strain: $\pm 600 \mathrm{ppm}$. Additionally, it might be necessary to include higher order terms in $F_{M, 1}$ for the analysis of experimental results when $B_{2 \perp}, B_{4 / /}$, and $B_{4 \perp}$ are close to zero. For the range $y=0-7 \%$ an almost linear decrease of $B_{2 \perp}$ is evidenced. For larger concentrations $7.0 \%<y<11.3 \%, B_{2 \perp}$ depends less strongly on $y$. This result can be explained by the behavior of Luttinger parameters in the range $7.0 \%<y<11.3 \%$ and the lower Fermi energy for $y=11.3 \%$.

The fourth-order parameters $B_{4 / /}$ and $B_{4 \perp}$ are presented in Figs. $7(\mathrm{~b})$ and $7(\mathrm{c})$, respectively. We can see that the experimental data of $B_{4 / /}$ are very well reproduced by the microscopic calculations. The $B_{4 / /}$ values obtained using the the 40-band k.p model in $(\mathrm{Ga}, \mathrm{Mn})(\mathrm{As}, \mathrm{P})$ are in much better agreement with experimental values than in ternary (Ga,Mn)As using the 6-band k.p model ${ }^{9}$. We believe that the more accurate description of the MA originates from a better description of the bands with our 40-band k.p model in the range used for the calculation of the MA, in particular from a good determination of the Luttinger parameters. Besides, the assumption that consists in taking the Fermi level as a constant can be considered as relevant because of the almost perfect agreement between experiment and theory. 
Unlike the results of $B_{4 / /}$, those of $B_{4 \perp}$ (Fig. $7(\mathrm{c})$ ) show large variations between the microscopic values and the experimental ones. This discrepancy becomes less important for samples which present close experimental values of $B_{4 / /}$ and $B_{4 \perp}$ (samples $\mathrm{B}, \mathrm{D}, \mathrm{E}$, and $\mathrm{G}$ ). However, the predictions of microscopic calculations could not explain the large variation observed in samples A, C and F. Moreover, we can notice that the change of sign for $B_{4 \perp}$ is not reproduced by microscopic calculations. The same problem has also been reported in Ref. 9 for $\mathrm{Ga}_{1-x} \mathrm{Mn}_{x}$ As. At the moment, the reason for this discrepancy is not yet understood. We can not exclude the possibility that the change of sign of $B_{4 \perp}$ is an artifact of the experimental method for determining $B_{4 \perp}{ }^{9,44}$. In samples with high $B_{2 \perp}$ coefficient the experimental determination of $B_{4 \perp}$ might be unreliable ${ }^{10}$.

\section{CONCLUSION}

To summarize, we have presented a theoretical survey of the magnetic properties of ferromagnetic $\mathrm{Ga}_{1-x} \mathrm{Mn}_{x} \mathrm{As}_{1-y} \mathrm{P}_{y} / \mathrm{GaAs}$ epilayers using the mean-field approximation and the 40-band k.p model, in which the band structure and band parameters are calculated. The present k.p description accurately reproduces the overall band diagram, as well as the band shifts and the carrier effective masses versus phosphorus content. We have considered the effect of the phosphorus composition on the MA. The present microscopic model has been validated through an accurate set of comparisons with experimental results. In particular, the values of $B_{2 \perp}$ and $B_{4 / /}$ anisotropy parameters are very well reproduced.

In the next step, it would be instructive to examine the effect of the introduction of shear deformation in our microscopic calculations. Firstly, the anisotropy coefficient $\mathrm{B}_{2 \|}$, which may be associated with magnetostriction or may result from anisotropic Mn distribution $^{6,10,52}$, plays an important role in the orientation of the in-plane magnetic easy axes. This in-plane uniaxial MA has the same symmetry as the one that would arise from an $\epsilon_{x y}$ shear strain although never evidenced experimentally. Secondly, the recent demonstration of magnetization precession triggered by quasi-transverse picosecond strain pulses emphasizes the importance of shear deformation for magnetization manipulation ${ }^{55}$.

The 40-band k.p model also offers the possibility to calculate the optical properties, in particular the dielectric permittivity and the spectral dependence of the magneto-optical Kerr effect, which is of great importance for the optimization of optical pump-probe exper- 
iments and magneto-optical imaging of magnetic domains in these ferromagnetic semiconductors.

\section{ACKNOWLEDGEMENTS}

The authors thank Laura Thevenard from INSP for useful discussions. This work was performed in the framework of the MANGAS project (ANR 2010-BLANC-0424-02).

* kais.boujdaria@fsb.rnu.tn

$\dagger$ Current adress: Laboratoire Nanostructures et Magnétisme, INAC, CEA-Grenoble, 17, rue des Martyrs 38054 Grenoble cedex 9, France

1 H. Ohno, Science 281, 951 (1998).

2 T. Dietl, H. Ohno, F. Matsukura, J. Cibert, and D. Ferrand, Science 287, 1019 (2000).

3 S. A. Wolf, D. D. Awschalom, R. A. Buhrman, J. M. Daughton, S. von Molnar, M. L. Roukes, A. Y. Chtchelkanova, and D. M. Treger, Science 294, 1488 (2001).

4 T. Jungwirth, J. Mašek, J. Kucera, and A. H. MacDonald, Rev. Mod. Phys. 78, 809 (2006).

5 L. Chen, X. Yang, F. Yang, J. Zhao, J. Misuraca, P. Xiong, and S. von Molnar, Nano. Lett. 11, 2584 (2011).

6 M. Sawicki, K.-Y. Wang, K. W. Edmonds, R. P. Campion, C. R. Staddon, N. R. S. Farley, C. T. Foxon, E. Papis, E. Kamińska, A. Piotrowska, T. Dietl, and B. L. Gallagher, Phys. Rev. B 71, 121302(R) (2005).

7 Shinhee Kim, Hakjoon Lee, Taehee Yoo, Sangyeop Lee, Sanghoon Lee, X. Liu, and J. K. Furdyna, J. Appl. Phys. 107, 103911 (2010).

8 Kh. Khazen, H. J. von Bardeleben, J. L. Cantin, L. Thevenard, L. Langeau, O. Mauguin, and A. Lemaître, Phys. Rev. B 77, 165204 (2008).

9 M. Glunk, J. Daeubler, L. Dreher, S. Schwaiger, W. Schoch, R. Sauer, W. Limmer, A. Brandlmaier, S. T. B. Goennenwein, C. Bihler, and M. S. Brandt, Phys. Rev. B 79, 195206 (2009).

10 J. Zemen, J. Kucera, K. Olejnik, and T. Jungwirth, Phys. Rev. B 80, 155203 (2009).

11 G. V. Astakhov, A. V. Kimel, G. M. Schott, A. A. Tsvetkov, A. Kirilyuk, D. R. Yakovlev, G. Karczewski, W. Ossau, G. Schmidt, L. W. Molenkamp, and Th. Rasing, Appl. Phys. Lett. 86, 
$152506(2005)$.

12 J. Wunderlich, A. C. Irvine, J. Zemen, V. Holý, A. W. Rushforth, E. De Ranieri, U. Rana, K. Výborný, Jairo Sinova, C. T. Foxon, R. P. Campion, D. A. Williams, B. L. Gallagher, and T. Jungwirth, Phys. Rev. B 76, 054424 (2007).

13 T. Dietl, H. Ohno, and F. Matsukura, Phys. Rev. B 63, 195205 (2001).

14 M. Abolfath, T. Jungwirth, J. Brum, and A. H. MacDonald, Phys. Rev. B 63, 054418 (2001).

15 X. Liu, W. L. Lim, L. V. Titova, M. Dobrowolska, J. K. Furdyna,M. Kutrowski, and T. Wojtowicz, J. Appl. Phys. 98, 063904 (2005).

16 L. Thevenard, L. Largeau, O. Mauguin, A. Lemaître, Phys. Rev. B 75, 195218 (2007).

17 A. Dourlat, V. Jeudy, C. Testelin, F. Bernardot, K. Khazen, C. Gourdon, L. Thevenard, L. Largeau, O. Mauguin, and A. Lemaî tre, J. Appl. Phys. 102, 023913 (2007).

18 L. Thevenard, L. Largeau, O. Mauguin, G. Patriarche, A. Lemaître, N. Fernier, and J. Ferré, Phys. Rev. B 73, 195331 (2006).

19 A. W. Rushforth, E. De Ranieri, J. Zemen, J. Wunderlich, K. W. Edmonds, C. S. King, E. Ahmad, R. P. Campion, C. T. Foxon, B. L. Gallagher, K. Výborný, J. Kucera, and T. Jungwirth, Phys. Rev. B, 78, 085314 (2008).

20 M. Overby, A. Chernyshov, L. P. Rokhinson, X. Liu, and J. K. Furdyna, Appl. Phys. Lett. 92, 192501 (2008).

21 A. Casiraghi, A. W. Rushforth, J. Zemen, J. A. Haigh, M. Wang, K. W. Edmonds, R. P. Campion and B. L. Gallagher, Appl. Phys. Lett. 101, 082406 (2012)

22 J. Wenisch, C. Gould, L. Ebel, J. Storz, K. Pappert, M. J. Schmidt, C. Kumpf, G. Schmidt, K. Brunner, and L. W. Molenkamp, Phys. Rev. Lett. 99, 077201 (2007).

23 A. V. Scherbakov, A. S. Salasyuk, A. V. Akimov, X. Liu, M. Bombeck, C. Brüggemann, D. R. Yakovlev, V. F. Sapega, J. K. Furdyna, and M. Bayer, Phys. Rev. Lett. 105, 117204 (2010).

24 M. Bombeck, A. S. Salasyuk, B. A. Glavin, A. V. Scherbakov, C. Brüggemann, D. R. Yakovlev, V. F. Sapega, X. Liu, J. K. Furdyna, A. V. Akimov, and M. Bayer, Phys. Rev. B 85, 195324 (2012).

25 A. Lemaître, A. Miard, L. Travers, O. Mauguin, L. Largeau, C. Gourdon, V. Jeudy, M. Tran, and J.-M. George, Appl. Phys. Lett. 93, 021123 (2008).

26 M. Cubukcu, H. J. von Bardeleben, Kh. Khazen, J. L. Cantin, O. Mauguin, L. Largeau, and A. Lemaître, Phys. Rev. B 81, 041202 (2010). 
27 P. R. Stone, K. Alberi, S. K. Z. Tardif, J. W. Beerman, K. M. Yu, W. Walukiewicz, and O. D. Dubon, Phys. Rev. Lett. 101, 087203 (2008).

28 S. Haghgoo, M. Cubukcu, H. J. von Bardeleben, L. Thevenard, A. Lemaître, and C. Gourdon, Phys. Rev. B 82, 041301 (R) (2010).

29 J. Masek, J. Kudronovsky, F. Maca, J. Sinova, A. H. Macdonald, R. P. Campion, B. L. Gallagher, and T. Jungwirth, Phys. Rev. B 75, 045202 (2007).

30 L. Thevenard, E. Peronne, C. Gourdon, C. Testelin, M. Cubukcu, E. Charron, S. Vincent, A. Lemaître, and B. Perrin, Phys. Rev. B 82, 104422 (2010).

31 L. Thevenard,S. A. Hussain, H. J. von Bardeleben, M. Bernard, A. Lemaître, and C. Gourdon, Phys. Rev. B 85, 064419 (2012).

32 M. Yahyaoui, C. Testelin, C. Gourdon, and K. Boujdaria, J. Appl. Phys. 111, 033902 (2012).

33 I. Saïdi, S. Ben Radhia, and K. Boujdaria, J. Appl.Phys. 107, 043701 (2010).

34 R. Neffati, I. Saïdi, and K. Boujdaria, J. Appl.Phys. 112, 053716 (2012).

35 G. E. Pikus and G. L. Bir, Fiz. Tverd. Tela (Leningrad) 1 (1959) 1642 Sov. Phys. Solid. State 1, 1502 (1960); see also, G. L. Bir and G. E. Pikus, Symmetry and Strain-Induced Effects in Semiconductors (Wiley, New-York, 1974).

36 E. O. Kane, in Handbook on Semiconductors, edited by T. S. Moss (North-Holland, Amesterdam, 1982), p. 193.

37 T. B. Bahder, Phys. Rev. B 41, 11992 (1990).

38 San-Guo Shenyz and Xi-Qing Fanz, J. Phys.: Condens. Matter. 8, 4369 (1996).

39 J. A Gaj, R. Planel, and G. Fishman, Sol. St. Commun. 29, 435 (1979).

40 F. Glas, G. Patriarche, L. Largeau, and A. Lemaître, Phys. Rev. Lett. 93, 086107 (2004).

41 Jiseok Kim and Massimo V. Fischetti, J. Appl. Phys. 108, 013710 (2010).

42 R. Enderlein, G. M. Sipahi, L. M. R. Scolfaro and J. R. Leite. Phys. Stat. Sol(b) 206, 623 (1998).

43 I. Vurgaftman, J. R. Meyer, and L. R. Ram-Mohan, J. Appl. Phys. 89, 5815 (2001).

44 X. Liu and K. Furdyna, J. Phys. Condens. Matter 18, R245 (2006).

45 W. Limmer, M. Glunk, J. Daeubler, T. Hummel, W. Scoch, R. Sauer, C. Bihler, H. Huebl, M. S. Brandt, and S. T. B. Goennenwein, Phys. Rev. B 74, 205205 (2006).

46 O. M. Fedorych, E. M. Hankiewicz,Z. Wilamowski, and J. Sadowski, Phys. Rev. B 66, 045201 (2002). 
47 C. Bihler, G. Ciatto, H. Huebl, G. Martinez-Criado, P. J. Klar, K. Volz, W. Stolz, W. Schoch, W. Limmer, F. Filippone, A. Amore Bonapasta, and M. S. Brandt, Phys Rev B 78, 235208 (2008).

48 Zheng Wen-Chen, Physica B 179, 1971992

49 U. Welp, V. K. Vlasko-Vlasov, A. Menzel, H. D. You, X. Liu, J. K. Furdyna, and T. Wojtowicz, Appl. Phys. Lett. 85, 260 (2004).

50 K.Y. Wang, M. Sawicki, K.W. Edmonds, R.P. Campion, S. Maat, C.T. Foxon, B.L. Gallagher, T. Dietl, Phys. Rev. Lett. 95 (2005) 217204.

51 S. Piano, X. Marti, A. W. Rushforth, K. W. Edmonds, R. P. Campion, M. Wang, O. Caha, T. U. Sch'ulli, V. Holý, and B. L. Gallagher, Appl. Phys. Lett. 98, 152503 (2011).

52 M. Birowska, C. Sliwa, J. A. Majewski, and T. Dietl, Phys. Rev. Lett. 108, 237203 (2012).

53 W. Stefanowicz, C. Śliwa, P. Aleshkevych, T. Dietl, M. Döppe, U. Wurstbauer, W. Wegscheider, D. Weiss, and M. Sawicki, Phys. Rev. B 81, 155203 (2010).

54 X. Liu, Y. Sasaki, and J. K. Furdyna, Phys. Rev. B 67, 205204 (2003).

55 M. Bombeck, J. V. Jäger, A. V. Scherbakov, T. Linnik, D. R. Yakovlev, X. Liu, J. K. Furdyna, A. V. Akimov, and M. Bayer, Phys. Rev. B 87, 060302(R) (2013). 


\section{FIGURES}

Figure 1 : Band structure of $\mathrm{Ga}_{0.9631} \mathrm{Mn}_{0.0369}$ As compound semiconductor along the high symmetry lines of the first Brillouin zone.

Figure 2: Magnified view of the heavy- and light hole bands and the split-off band in the vicinity of the $\mathrm{k}=0$ zone center, for samples with three different phosphorus fraction (a) $\mathrm{y}=0 \%$, (b) $\mathrm{y}=3.4 \%$, and (c) $\mathrm{y}=11.3 \%$. (d) Comparison of the 40-band (solid line) and 6-band (dashed line) k.p models for the $y=0 \%$ sample (Ga,Mn)As.

Figure 3 : Calculated band-gap energy $\mathrm{E}_{g}^{\Gamma}$ of $\mathrm{Ga}_{1-x} \mathrm{Mn}_{x} \mathrm{As}_{1-y} \mathrm{P}_{y}$ as a function of $y$ content for the samples of Table 1.

Figure 4 : The effective masses of (a) the electron $m_{e}\left(\Gamma_{6 C}\right),(\mathbf{b})$ heavy-hole and (c) light-hole for $\mathrm{Ga}_{1-x} \mathrm{Mn}_{x} \mathrm{As}_{1-y} \mathrm{P}_{y} / \mathrm{GaAs}$ calculated with k.p method.

Figure 5: Luttinger parameters $\gamma_{\ell}(\ell=1,2,3)$ of $\mathrm{Ga}_{1-x} \mathrm{Mn}_{x} \mathrm{As}_{s_{1-y}} \mathrm{P}_{y} / \mathrm{GaAs}$ obtained with 40-band k.p model (symbols). The full lines represent the Luttinger parameters for unstrained $\mathrm{GaAs}_{1-y} \mathrm{P}_{y}$ (from [43]).

Figure 6: $\Delta F_{M, 1}$ calculated as a function of the magnetization orientation and phosphorus concentration for $\mathbf{M}$ (a) in the (010) plane and (b) in the (100) plane. The results of the microscopic model are represented by the solid symbols. The solid lines are least-square fit curves..

Figure 7 : Phosphorus concentration dependence of (a) the uniaxial out-of-plane mag-

netic anisotropy constant $B_{2 \perp}$, (b) the in-plane cubic anisotropy constant $B_{4 / /}$ and (c) the out-of-plane cubic anisotropy constant $B_{4 \perp}$. The magnetic anisotropy constants obtained with microscopic calculations (circles) are compared with the experimental data obtained from FMR experiments at $\mathrm{T}=4.2 \mathrm{~K}$ (squares). 
TABLE I. $\mathrm{Ga}_{1-x} \mathrm{Mn}_{x} \mathrm{As}_{1-y} \mathrm{P}_{y}$ sample parameters: the phosphorus concentration [P], the lattice mismatch $l m$, the corresponding strain component $\epsilon_{z z}$, the saturation magnetization $\mathrm{M}_{S}$ at $\mathrm{T}=4$ $\mathrm{K}$, the effective Mn concentration $x_{e f f}$, and the splitting parameter of the valence subbands $B_{G}$.

\begin{tabular}{cccccccc}
\hline \hline Sample & $\mathrm{A}$ & $\mathrm{B}$ & $\mathrm{C}$ & $\mathrm{D}$ & $\mathrm{E}$ & $\mathrm{F}$ & $\mathrm{G}$ \\
\hline$[\mathrm{P}](\%)$ & 0 & 2.6 & 3.4 & 4.3 & 5.6 & 7 & 11.3 \\
$\operatorname{lm}(\mathrm{ppm})$ & 4990 & 280 & 0 & -1200 & -2320 & -3580 & -6620 \\
$\epsilon_{z z}(\%)$ & 0.23 & 0.013 & 0 & -0.055 & -0.11 & -0.16 & -0.31 \\
$\left.\mathrm{M}_{S}(\mathrm{emu} \mathrm{cm})^{-3}\right)$ & 37.6 & 37.7 & 37.3 & 40.75 & 42.6 & 39.2 & 35 \\
$x_{e f f}(\%)$ & 3.69 & 3.67 & 3.63 & 3.96 & 4.14 & 3.80 & 3.49 \\
$B_{G}(\mathrm{meV})$ & -26.0 & -26.3 & -26.0 & -28.5 & -30.0 & -27.5 & -24.7 \\
\hline \hline
\end{tabular}

TABLE II. Fermi level $E_{F}$, theoretical carrier density $p_{\text {theo }}$ and estimated carrier density $p_{\text {est }}$ of $\mathrm{Ga}_{1-x} \mathrm{Mn}_{x} \mathrm{As}_{1-y} \mathrm{P}_{y}$ samples.

\begin{tabular}{cccccccc}
\hline \hline Sample & $\mathrm{A}$ & $\mathrm{B}$ & $\mathrm{C}$ & $\mathrm{D}$ & $\mathrm{E}$ & $\mathrm{F}$ & $\mathrm{G}$ \\
\hline$E_{F}(\mathrm{meV})$ & -110 & -110 & -110 & -110 & -110 & -110 & -81 \\
$p_{\text {est }}\left(10^{20} \mathrm{~cm}^{-3}\right)$ & 4.5 & 4.4 & 4.3 & 5.4 & 6.0 & 4.9 & 3.5 \\
$p_{\text {theo }}\left(10^{20} \mathrm{~cm}^{-3}\right)$ & 4.7 & 3.3 & 3.2 & 4.8 & 6.3 & 4.5 & 3.5 \\
\hline \hline
\end{tabular}

